Case report

\title{
Duodenal polyposis, a rare manifestation of gastrointestinal portal hypertension
}

\author{
Maria-Alexandra Florea*,1, Angela-Ștefania Varban'1, Simona Costache1, Corina \\ Angelescu², Cosmin Ciora ${ }^{2}$
}

'Department of Pathology, Emergency University Hospital, Bucharest, Romania; ${ }^{2}$ Gastroenterology and Hepatology Center, Fundeni Clinical Institute, Bucharest, Romania

\begin{abstract}
Portal hypertension can affect the entire gastrointestinal tract, including the duodenum. Portal hypertensive duodenopathy may occur relatively rare in patients with portal hypertension secondary to cirrhosis or extrahepatic portal venous obstruction. We report the case of a 63-years-old female patient with cirrhosis who underwent an esophagogastroduodenoscopy. Multiple small duodenal polyps (2-3 mm) were found. The histopathologic examination of the duodenal biopsy specimen revealed a polypoid duodenal mucosa, with preserved villous architecture, with focal gastric foveolar metaplasia and numerous ectatic capillaries in lamina propria. The polypoid lesions found in the duodenum are a consequence of portal hypertension. The presence of one or several polyps in the duodenum of a patient with portal hypertension, with specific histological findings (dilated mucosal capillaries, no dysplasia) is diagnostic of duodenal polyp/polyposis in the context of portal hypertension.
\end{abstract}

Keywords: portal hypertension; cirrhosis; duodenal polyp; portal hypertensive duodenopathy

\section{Introduction}

Portal hypertensive duodenopathy (PHD) is one of the manifestations of portal hypertensive syndrome [1], causing duodenal varices, mucosal friability, erosions, ulceration, vascular ectasia, mosaic pattern and duodenal portal hypertensive polyp/polyposis, a rare finding that was recently described.

\section{Case report}

We report a case of a 63-year-old female patient with cirrhosis due to hepatitis virus $C$

Received: September 2019; Accepted after review: December 2019; Published: December 2019.

${ }^{*}$ Corresponding author: Maria Alexandra Florea, Pathology Department, Emergency University Hospital, Splaiul Independentei nr. 169, Bucharest, Romania Email: alexandra alex01@yahoo.com
(HVC) infection, who presented for an esophagogastroduodenoscopy. Her medical history includes sigmoid adenocarcinoma (status post proctosigmoidectomy 7 years), adjuvant chemotherapy and blood loss through colostomy (HGB $5 \mathrm{~g} / \mathrm{dL}$ ). The patient was diagnosed with cirrhosis in 2008. Abdominal ultrasound detected minimal ascitic fluid and hepatosplenomegaly. She was recently diagnosed with hepatic encephalopathy. Upper endoscopy revealed: grade I-II esophageal varices, severe portal hypertensive gastropathy, small, multiple duodenal polyps - 2-3 $\mathrm{mm}$, located in the duodenal bulb and second part of the duodenum (Figure 1). Two biopsies were taken.

Microscopically, the polypoid duodenal mucosa presented preserved villous architecture, with some enlarged villi (Figure 2), foveolar metaplasia of the surface epithelium and numerous ectatic and 
congested capillaries in the lamina propria (Figure 3). There was no inflammation or epithelial dysplasia.

Immunohistochemistry tests were performed: CD34 and D2-40 were positive in the endothelium of proliferating blood vessels and lymphatics in the lamina propria (Figure
4). Ki 67 proliferation index was $<1 \%$ (Figure 5).

Based on the clinical information, correlated with histopathological and immunohistochemical findings, a diagnosis of duodenal portal hypertensive polyp/polyposis was established.

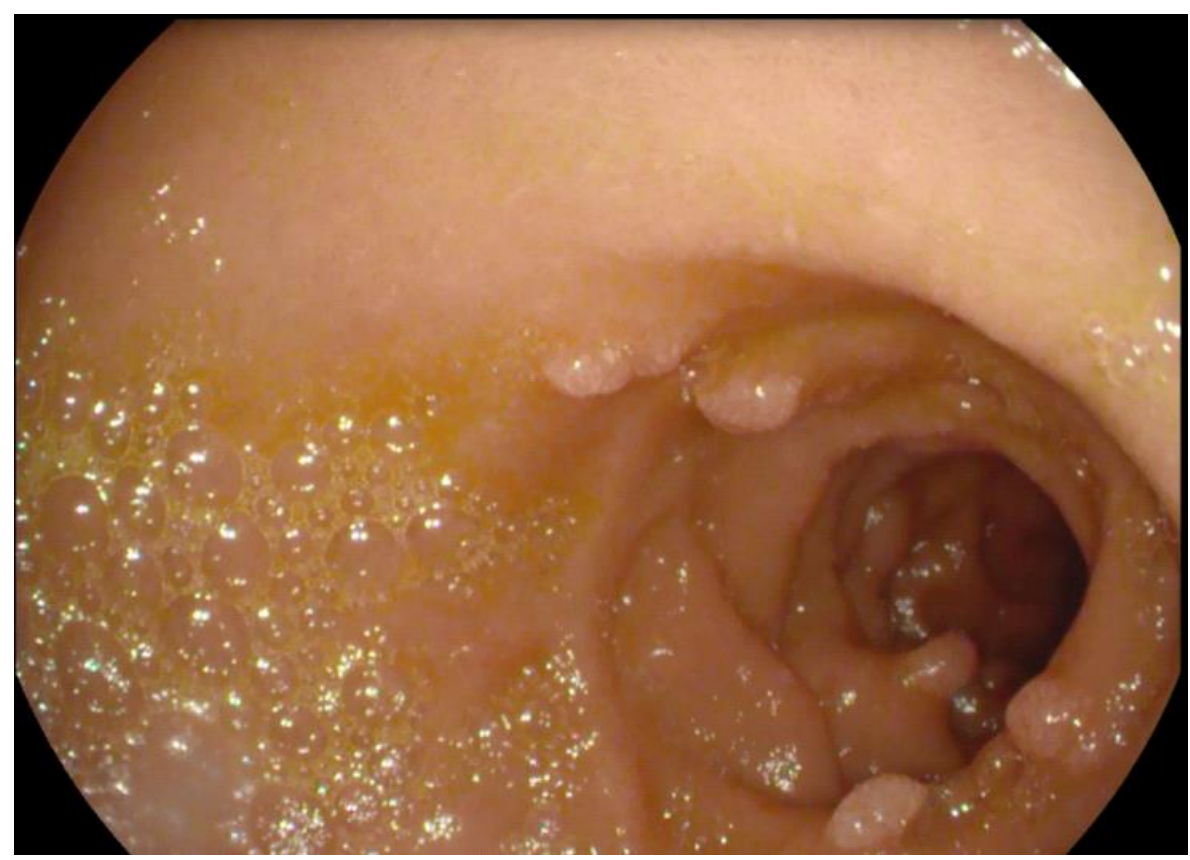

Fig. 1. Endoscopic image showing small sessile polyps $(2-3 \mathrm{~mm})$, located in the duodenal bulb and second part of the duodenum. Courtesy of ENGIP

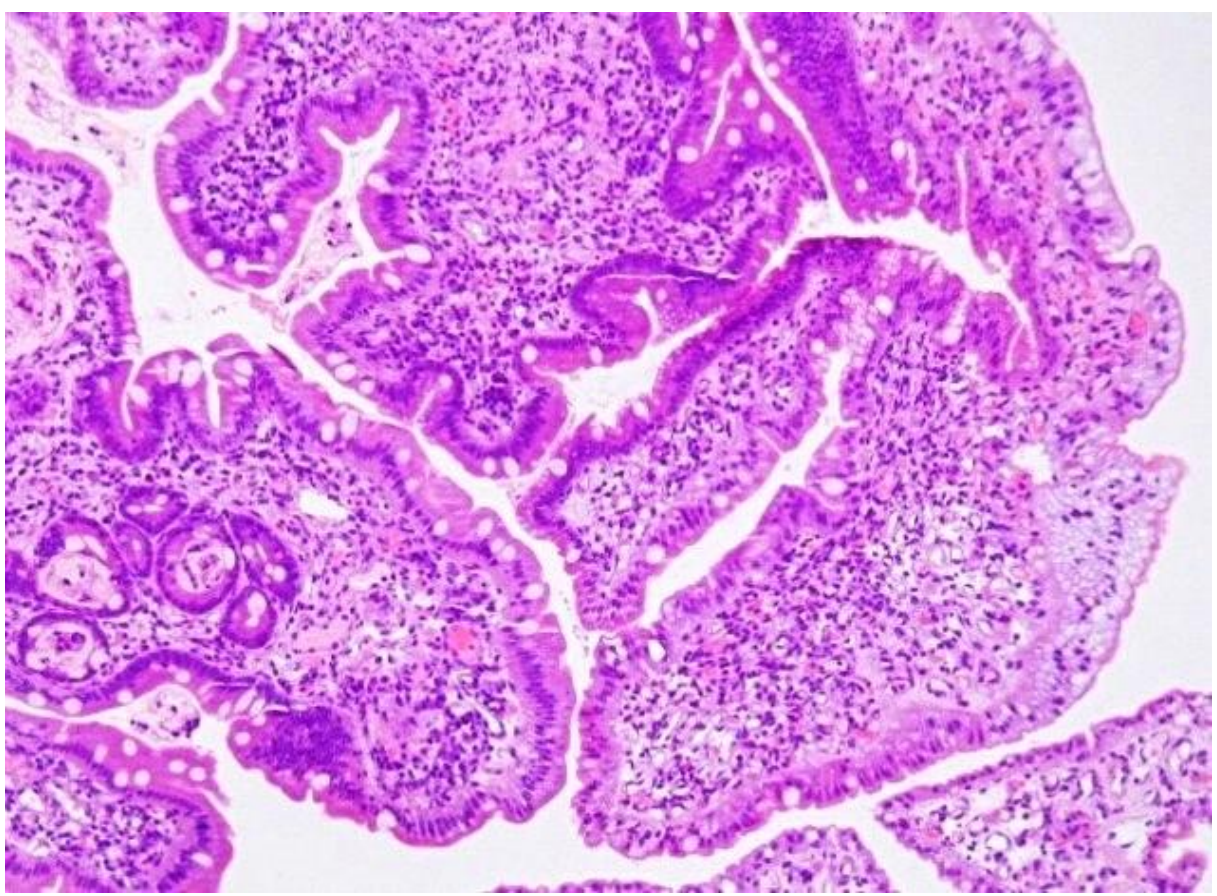

Fig. 2. Duodenal mucosa with preserved villous architecture, with some enlarged villi. There is no pathologic inflammation and no epithelial dysplasia (HE, x100). Courtesy of ENGIP. 


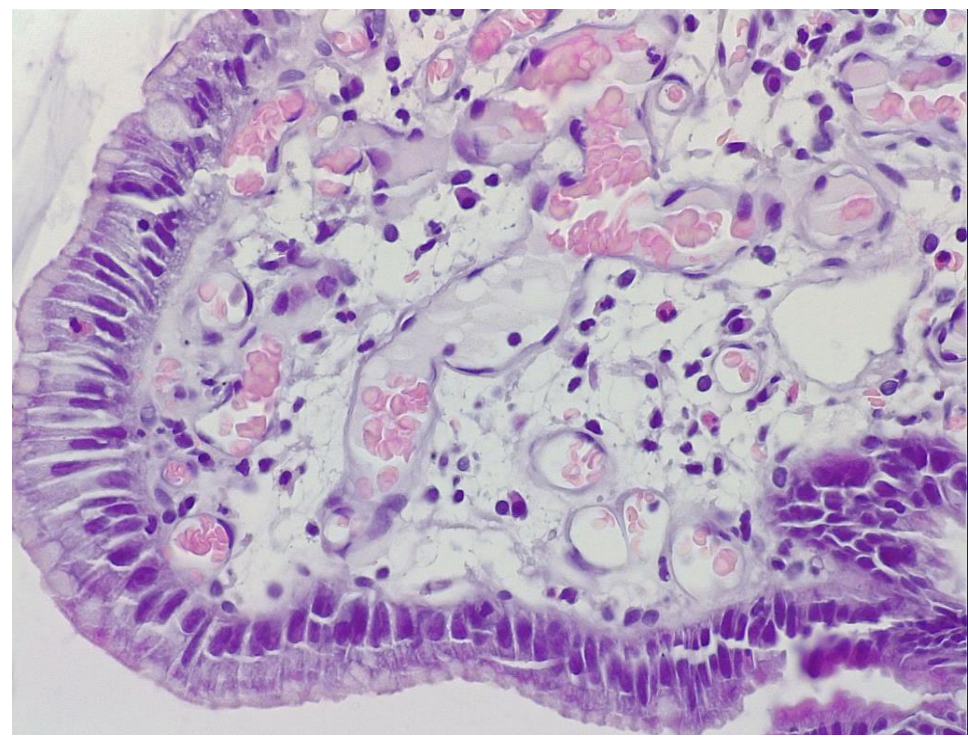

Fig. 3. Numerous ectatic capillaries in lamina propria. Gastric foveolar metaplasia of the surface duodenal epithelium. (HE, x200). Courtesy of ENGIP.

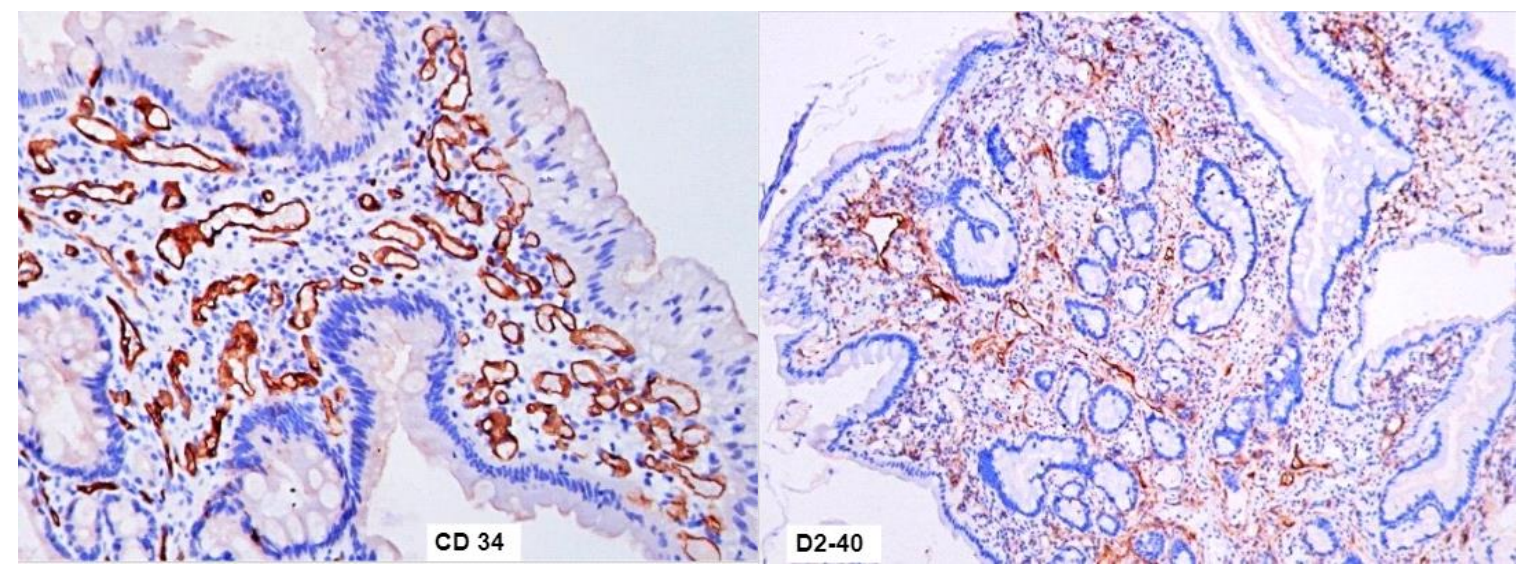

Fig. 4. Endothelial cells of blood and lymphatic vessels were positive for CD 34 and D2-40, respectively (IHC, anti CD34 Ab and anti D2-40 Ab, x100). Courtesy of ENGIP.

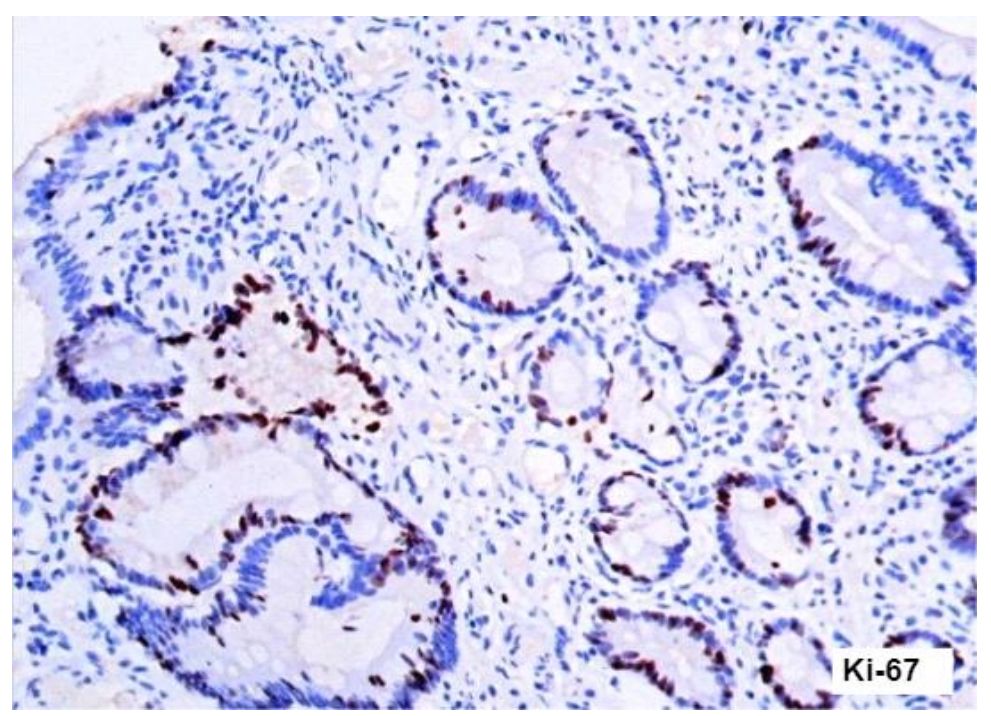

Fig. 5. Low proliferation index (Ki 67), predominantly in the duodenal crypts (IHC, anti-Ki67 Ab, x200). Courtesy of ENGIP. 


\section{Discussions}

The abnormal blood pressure in the portal venous system is due to chronic end-stage liver disease or to extrahepatic portal venous obstruction. Gastrointestinal manifestation of portal hypertension include esophageal varices, gastric varices in cardia and fundus, portal hypertensive gastropathy in body and fundus, GAVE (gastric antral vascular ectasia), portal hypertensive enteropathy, portal hypertensive colopathy [2].

Portal hypertensive duodenopathy is characterized by patchy or diffuse congestion of the duodenal mucosa, mosaic pattern, edema, friability, erosions or ulcerations, duodenal varices [3, 4]. Portal hypertensive duodenopathy has been described in $8.4 \%$ [1], respectively in $51.4 \%$ [5] of patients with portal hypertension.

Duodenal polyps are a rare manifestation of PHD and have been described in reports [6$10]$ and recent studies [11, 12]. Most cases presented as multiple polyps, ranging in size, from 1-2 $\mathrm{mm}$ [10] to $3 \mathrm{~cm}$ [6], located in first and second part of the duodenum, some of them being responsible for gastrointestinal bleeding. There was no gender predilection, age of patients ranging from 1 to 73 years old. Histological findings of the duodenal polyps described in the literature included: vascular ectasia/congestion/thrombi, gastric foveolar metaplasia, reactive nuclear atypia, fibrosis and smooth muscle proliferation.

Considering the patient's history of colonic adenocarcinoma, there was the assumption that the duodenal polyps found were adenomatous. Fortunately for the patient, no dysplasia was identified. Other differential diagnosis of duodenal portal hypertensive polyps includes: duodenal pancreatic or gastric heterotopia, duodenal hamartomatous polyps, inflammatory bowel disease associated inflammatory polyps and other lesions described in Table 1.

Table 1. Differential diagnosis of polyps located in the duodenum

\begin{tabular}{c|c}
\hline \multicolumn{2}{|c}{ Small intestine polyps } \\
\hline Proximal & Distal \\
\hline Adenoma & Carcinoid \\
Duodenitis & Lymphoma \\
\hline Lymphoid hyperplasia & GIST \\
\hline Pancreatic and gastric heterotopia & Adenocarcinoma \\
Brunner's gland hamartoma & Metastasis \\
Hamartomatous polyps & Melanoma \\
\hline
\end{tabular}

\section{Conclusions}

Duodenal polyps are a rare manifestation of portal hypertension and an accurate diagnosis should be made, based on the clinical context and typical microscopic findings: numerous ectatic mucosal capillaries and no dysplasia. Clinicians should keep in mind this type of non-neoplastic polyps when assessing patients with history of digestive adenocarcinoma and portal hypertension.

\section{Consent}

Written informed consent was obtained from the patient for publication of this case report.

\section{Competing interests}

The authors declare that they have no competing interests. 


\section{References}

1. Menchén L, Ripoll C, Marín-Jiménez I, et al. Prevalence of portal hypertensive duodenopathy in cirrhosis: clinical and haemodynamic features. Eur J Gastroenterol Hepatol 2006; 18(6):649-653.

2. Tang S-J. Portal hypertensive gastropathy, enteropathy, and colopathy. VJGIEN 2013; 1(1):269-271.

3. Gupta R, Saraswat VA, Kumar M, Naik SR, Pandey R. Frequency and factors influencing portal hypertensive gastropathy and duodenopathy in cirrhotic portal hypertension. $J$ Gastroenterol Hepatol 1996; 11(8):728-733.

4. Shudo R, Yazaki Y, Sakurai S, Uenishi H, Yamada H, Sugawara K. Duodenal erosions, a common and distinctive feature of portal hypertensive duodenopathy. $A m \quad J$ Gastroenterol 2002; 97(4):867-873.

5. Barakat M, Mostafa M, Mahran Z, Soliman AG. Portal hypertensive duodenopathy: clinical, endoscopic, and histopathologic profiles. $A m \mathrm{~J}$ Gastroenterol 2007; 102(12):2793-2802.

6. Zeitoun JD, Chryssostalis A, Terris B, Prat F, Gaudric M, Chaussade S. Portal hypertensive duodenal polyp: a case report. World J Gastroenterol 2007; 13(9):1451-1452.
7. Pillai SB, Ram Ganesh VR, Mohanakrishnan A, Nirmala V. Portal duodenopathy presenting as polyposis. Indian J Pathol Microbiol 2010; 53(3):558-559.

8. Devadason D, Murphy MS, Brown R, Wilson D, McKiernan PJ. Duodenal capillary hemangiomatous polyps: a novel manifestation of extrahepatic portal hypertension? J Pediatr Gastroenterol Nutr 2007; 45(1):114-116.

9. Lemmers A, Evrard $S$, Demetter $P$, et al. Gastrointestinal polypoid lesions: a poorly known endoscopic feature of portal hypertension. United European Gastroenterol J 2014; 2(3):189-196.

10. Gurung A, Jaffe $P$, Zhang $X$. Duodenal polyposis secondary to portal hypertensive duodenopathy. World J Gastrointest Endosc 2015; 7(17):1257-1261.

11. Aravind $C$, Arul Murugan $S$, Sai Ravi Kiran B. A study of portal hypertensive polyps. Int $J A d v$ Med 2017; 4(1):88-91.

12. Kara $D$, Hüsing-Kabar $A$, Schmidt $H$, et al. Portal Hypertensive polyposis in advanced liver cirrhosis: the unknown entity? Canadian J Gastroenterol Hepatol 2018; Article ID 2182784, 7 pages, 2018. 